Jack $A$. Wilkie and Knut Moeller

\title{
Nonlinearity of Magnetostrictive Torque Sensor under Varying External Magnetic Field Strength
}

\begin{abstract}
Correctly torquing bone screws is an important factor in achieving positive patient outcomes during orthopaedic surgery. A torque-limiting smart screwdriver concept has been proposed, and ongoing work is being undertaken to model the screwing process and allow the concept to work. These models require experimental validation, so a test rig was developed. The magnetostrictive torque sensor in this test rig was affected by magnetic parts of the test rig, which offset the zero-torque point; this raised concerns over the effects on linearity, which were tested here. The torque sensor was tested against a non-magnetostrictive reference under varying external magnetic conditions. While the magnetic field offset the torque, there was no notable change in linearity under the conditions tested, and the linearity was always within the datasheet specifications. Hence, we conclude that in the context of this test rig, there were no negative effects on linearity, although under higher loading or stronger magnetic conditions, this may not hold.
\end{abstract}

Keywords: Magnetostrictive, Torque sensor, Torque measurement, Nonlinearity, Linearity, Magnetic field

https://doi.org/10.1515/cdbme-2021-2194

\section{Introduction}

Bone screws are widely used in orthopaedic surgery to secure implants, and to support bones during natural healing. In both cases, it is critical that the connections are as resilient as possible. The correct torquing of these screws is a major factor in the success of the joint, as over torquing will strip the threads and compromise strength [1], and under-torqued screws are susceptible to loosening over time [2]. While surgeons are highly skilled, it is easy to misjudge the torque, or incorrectly torque a screw [3].

Wilkie et al. [4] proposed the use of a smart screwdriver to automatically identify and enforce the optimal torque. Previous work has focused on developing and testing models of screw insertion for detecting the bone material properties [4]-[6], and for predicting the optimal torque from these properties [7]. To test these models, a test rig was developed [8]. The test rig had to collect torque and displacement data with a high accuracy and sample rate.

The test rig uses a rotational torque sensor (NCTE 230020-1-AU-0-0, NCTE AG) to measure to torque in the rotating screw shaft. This sensor uses contactless inversemagnetostrictive technology to measure torque [9]; while effective, it was noted that the indicated torque value drifted when external magnetic effects were introduced, in the absence of any changes to actual torque. These external magnetic effects came from the stepper motor, which contains a magnetic rotor, and the screwdriver bit holder, which contains a small magnet to retain the screwdriver bit; hence, it was not possible to simply remove these effects. These effects could also be minimised by increasing the separation between the sensor and magnetic sources (which was possible due to the paramagnetic aluminium couplers that did not couple the magnetic fields), but only to a degree.

While it was clear from preliminary tests that the external magnetic fields introduced a zero-offset to the torque measurements, which could be compensated for with a simple re-zero, it required further testing to see exactly if/how the magnetic fields affected linearity, which would require a more complex compensation method. This paper investigates the effects of external magnetic fields on the linearity of the torque sensor used in our test rig. 


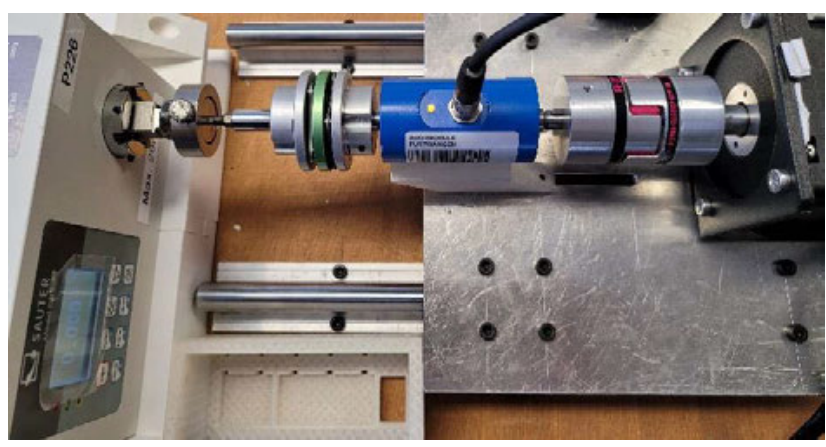

Figure 1: Test rig setup for testing magnetostrictive torque sensor (Right) against reference torque sensor (Left).

\section{Methods}

\subsection{Data Collection}

To collect data, a modified version of the test rig from [8] was used, shown in Fig. 1. To check the torque curves, we used a stationary torque sensor (Sauter DB 20-3, KERN \& SOHN $\mathrm{GmbH}$ ) as a reference. As this reference sensor did not use magnetostrictive technology, it should not be influenced by the magnetic field like sensor being tested.

The reference sensor was secured against the base of the test rig and the sample clamp. An adaptor was used to couple the screw bit holder on the test rig to the reference sensor. Both the reference sensor, and the test rig sensor were interfaced with a custom PC program to record synchronised data.

The magnetic field on the sensor was changed by adjusting the spacing between the magnetic screw-bit holder and torque sensor shaft by clamping the shaft coupler in three different positions as shown in Fig. 2. This changes the airgap and amount of magnetic coupling between the magnetic screw-bit holder and torque sensor shaft (Due to the low magnetic susceptibility of aluminium, the effects of coupling through the aluminium coupler can be neglected, however there may be some small influence from the steel screws and flexible elements).

To compare the torque curve of the sensor against the reference, the torque was ramped while continuously recording data from both sensors. The closed-loop stepper motor allowed a positioning resolution of $1 / 4000$ of a revolution, however, because of the stiffness of the connection between the torque sensors, only about 30 steps of motion were possible between zero torque and the maximum torque of the motor. The motor was rotated at 1 step per second for 40 s to fully tighten then loosen for each test. Data was recorded at $10 \mathrm{~Hz}$. This was repeated 3 times for the maximum spacing case, and 4 times each for the middle and minimum spacing cases.

\subsection{Data Analysis}

As the motor took a short time to stabilise into its new position after every step, the raw data contained a lot of torque oscillations, and therefore needed pre-processing before further analysis could be performed. Additionally, the reference torque sensor had a large dead zone of about $0.5 \mathrm{Nm}$.

Firstly, the data was trimmed to the period between when the reference sensor first read above $0 \mathrm{Nm}$, and when the maximum torque was first achieved. The stepping nature of the tightening resulted in the torque dropping then recovering to the correct level for each step; to compensate for this, the peak torque for each step was selected, and the dataset was reduced to contain only the samples that contained these peaks.

From this point, the torques from the sensor under test were plotted against the corresponding torques from the reference sensor. All curves were plotted on the same axes to show trends as spacing changed. The maximum nonlinearity was then calculated as the half of the range of deviation from the reference sensor, scaled to the maximum torque of the sensor and converted to a percentage:

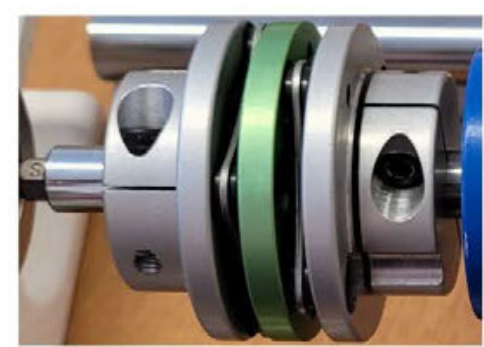

Minimum Dist.

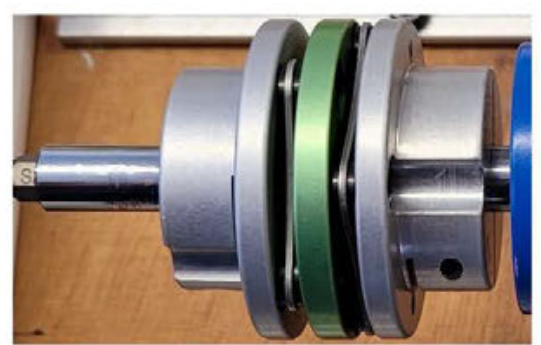

Middle Dist.

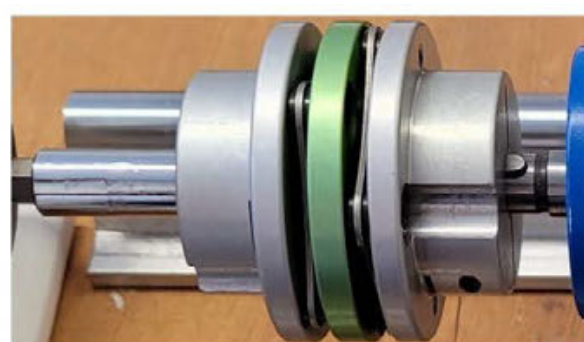

Maximum Dist.

Figure 2: Distances between screw bit holder and torque sensor for the 3 cases tested. 


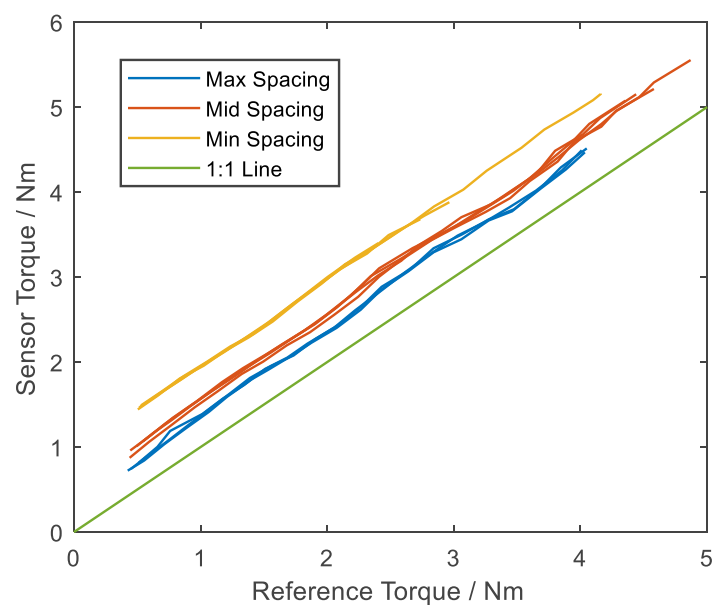

Figure 3: Torque curves for the different sensor spacing without zero-point correction.

$$
\frac{\left|\max \left(T_{\text {sensor }}-T_{\text {ref }}\right)-\min \left(T_{\text {sensor }}-T_{\text {ref }}\right)\right|}{2} \times \frac{100}{T_{\max }}
$$

\section{Results}

Figure 3 shows the torque curves for the sensor referenced against the reference sensor. Figure 4 also show the curves, but with the torque zero-point corrected using the first sample from the data which is always in a fully unloaded state. The torque zero-offset (mean \pm standard deviation) (related to the strength of magnetic influence) for the minimum spacing was $0.85 \pm 0.02 \mathrm{Nm}$, for the middle spacing was $0.56 \pm 0.00 \mathrm{Nm}$, and for the maximum spacing was $0.43 \pm 0.00 \mathrm{Nm}$. The maximum nonlinearity (mean \pm standard deviation) for the minimum spacing was $0.24 \pm 0.02 \%$, for the middle spacing was $0.53 \pm 0.05 \%$, and for the maximum spacing was $0.46 \pm 0.05 \%$.

\section{Discussion}

Figure 3 shows that the curves from all 3 cases tested are mostly parallel, with higher offsets when the spacing is lower and the magnetic influence is therefore higher, as expected from initial observations. Figure 4 shows the lines with the torque zero-offset corrected; in this case, the lines appear to mostly overlap closely enough that it is difficult to visually conclude whether the nonlinearity is equivalent.

The calculated average maximum nonlinearities were all at or below $0.5 \%$, which is the maximum allowed according to the datasheet specifications [10]; in this sense, the sensor is

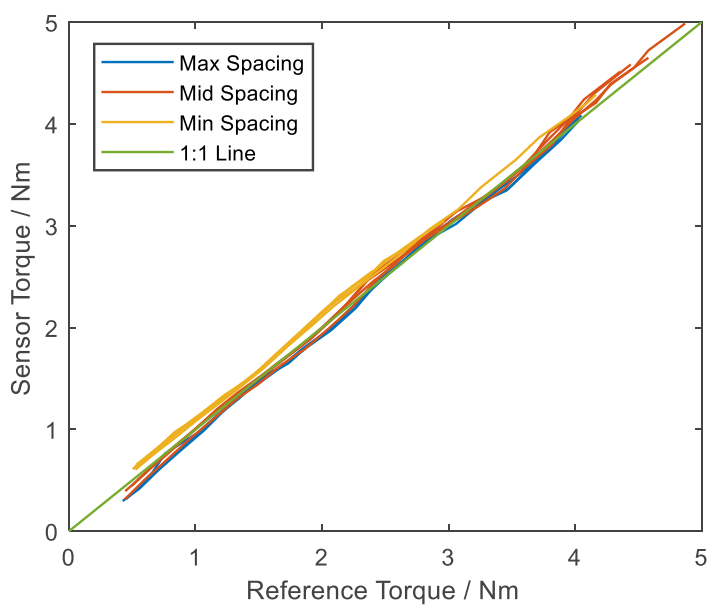

Figure 4: Torque curves for the different sensor spacing with zero-point correction.

operating fully within specification for the torque range tested, even at the maximum external magnetic influence levels tested here. This suggest that the only effect of the external magnetic influence is the zero-torque offset, and linearity is uncompromised. It is also noteworthy that the best nonlinearity was found at the highest level of magnetic influence, although this could be an artefact of the data processing employed.

Only the range of $0-5 \mathrm{Nm}$ was tested due to motor limitations, and given that the sensor range is $0-20 \mathrm{Nm}$, it is possible that further non-linearities may occur outside of the tested range, and these may exceed the specification; countering this, there is no obvious trend in the data that suggests extrapolation would result in higher nonlinearity. Also, for the application of testing bone screw models, it is unlikely that torques above $5 \mathrm{Nm}$ will be used, so this is not as important; however, for the generally applicability of this research, this is a noteworthy limitation. This could be addressed in future research with a more powerful motor, or the use of a gearbox, both of which would allow higher torques to be tested.

Due to the stiffness of the couplings between the two sensors and the motor, only a limited number of motor steps were possible between zero and full torque. Adding an elastic coupling or torsional spring would allow a higher resolution torque curve.

In this testing, the minimum offset torque was $0.43 \mathrm{Nm}$ for the maximum spacing tested. This case therefore still had external magnetic effects acting on the sensor (in isolation, the sensor was found to be within $0.1 \mathrm{Nm}$ of zero). This was mostly from the stepper motor, as the spacing on the motor side of the sensor was not adjusted during the experiment due to practical and time limitations. To test a larger range of 
magnetic influence strengths, the spacing between the sensor and motor should have also been varied. However, this would only be testing lower magnetic influences, which would be expected to have even less effect on the sensor and its nonlinearity, and it is unlikely that it would result in cases with worse nonlinearity.

In this testing, the quantitative strength of the magnetic field could not be measured due to a lack of suitable equipment. This also means that the maximum magnetic field tested is unknown. With appropriate information about the internal design of the sensor, it may be possible to determine the total magnetic flux coupled into the sensor from the torque offsets. It is also possible that stronger magnetic fields may result in measurable nonlinearities. Future work in this area should attempt to quantify the magnetic field strength/magnetic flux, and document any limits before linearity is affected, to provide solid recommendations for the design of systems involving magnetostrictive torque measurement.

This testing only considers the effects of static magnetic fields. If exposed to dynamic magnetic fields, the effects on the sensor could be different. At minimum, a changing magnetic field would be expected to modulate the torque signal as it shifts the zero point over time. Additionally, a changing magnetic field may induce currents in the internal electronics of the sensor, further interfering with the readings.

\section{Conclusion}

A magnetostrictive torque sensor was tested to determine how coupled external magnetic fields affected the linearity of the torque readings. The sensor was compared against a reference over a sweep of applied torque values in 3 configurations that applied different strengths of external magnetic fields to the sensor. While the zero point of the readings shifted as the applied magnetic strength changed, which can be corrected with a simple re-zeroing, there was no significant non-linearity detected due to the external magnetic field, and the nonlinearity was within the specifications from the datasheet. Further testing with higher torques may be able to cover more of the sensors range, as only $5 \mathrm{Nm}$ of the $20 \mathrm{Nm}$ range was able to be tested with the motor used here. Further work may also be able to test with stronger magnetic fields, as only a limited range of magnetic effects was tested here, and the magnetic field strengths were not quantified. Regardless, as the conditions here match those used for our model testing, we can conclude that external-magnetic-field-induced nonlinearity has not been compromising our experimental testing.

\section{Author Statement}

Research funding: This work was partially supported by grants "CiD" and "Digitalisation in the OR" from BMBF (Project numbers 13FH5E02IA and 13FH5I05IA). Conflict of interest: Authors state no conflict of interest.

\section{References}

[1] A. Feroz Dinah, S. C. Mears, T. A. Knight, S. P. Soin, J. T. Campbell, and S. M. Belkoff, 'Inadvertent Screw Stripping During Ankle Fracture Fixation in Elderly Bone', Geriatr. Orthop. Surg. Rehabil., vol. 2, no. 3, pp. 86-89, May 2011, doi: $10.1177 / 2151458511401352$.

[2] M. Evans, M. Spencer, Q. Wang, S. H. White, and J. L. Cunningham, 'Design and testing of external fixator bone screws', J. Biomed. Eng., vol. 12, no. 6, pp. 457-462, Nov. 1990, doi: 10.1016/0141-5425(90)90054-Q.

[3] M. J. Stoesz, P. A. Gustafson, B. V. Patel, J. R. Jastifer, and J. L. Chess, 'Surgeon Perception of Cancellous Screw Fixation', J. Orthop. Trauma, vol. 28, no. 1, p. e1, Jan. 2014, doi: 10.1097/BOT.0b013e31829ef63b.

[4] J. Wilkie, P. D. Docherty, and K. Möller, 'A simple screwing process model for bone material identification', Proc. Autom. Med. Eng., vol. 1, no. 1, Art. no. 1, Feb. 2020, doi: 10.18416/AUTOMED.2020.

[5] J. Wilkie, P. D. Docherty, and K. Möller, 'Developments in Modelling Bone Screwing', Curr. Dir. Biomed. Eng., vol. 6, no. 3, pp. 111-114, Sep. 2020, doi: 10.1515/cdbme-2020-3029.

[6] J. Wilkie, P. D. Docherty, and K. Möller, 'Model-based bone material property identification', - Autom., vol. 68 , no. 11 , pp. 913-921, Nov. 2020, doi: 10.1515/auto-2020-0083.

[7] J. Wilkie, P. D. Docherty, and K. Möller, 'Stripping Torque Model for Bone Screws', presented at the 11th IFAC Symposium on Biological and Medical Systems, Ghent, Belgium, In press.

[8] J. A. Wilkie and K. Möller, 'Test rig for bone screw insertion modelling', presented at the 15th Interdisciplinary Symposium Automation in Medical Engineering (AUTOMED 2021)., Basel, Switzerland, 2021.

[9] H. Wakiwaka and M. Mitamura, 'New magnetostrictive type torque sensor for steering shaft', Sens. Actuators Phys., vol. 91, no. 1, pp. 103-106, Jun. 2001, doi: 10.1016/S09244247(01)00490-3.

[10] NCTE AG, 'Torque Sensor Series 2300 Instruction Manual and Data Sheet.' Sep. 2020. Accessed: Jul. 12, 2021. [Online]. Available: https://ncte.com/wpcontent/uploads/2020/09/Instruction_manual_data_sheet_s eries2300_EN.pdf 